\title{
Implementación del Modelo Pss2b en Simulink
}

\author{
García Hidalgo, Ernesto $^{1}$ (D) ; Pérez Martínez, Maykop $^{2 \text {, * (iD) }}$; Rodríguez Domínguez, Yandry² \\ ${ }^{1}$ Unión Eléctrica, Dirección técnica grupo de desarrollo. La Habana, Cuba. \\ ${ }^{2}$ Centro de investigaciones y pruebas electroenergéticas, Universidad tecnológica de la Habana, Cuba.
}

\begin{abstract}
Resumen: Este trabajo presenta un estudio de los diferentes tipos de estabilidad que están presentes en los Sistemas Eléctricos de Potencia (SEP) atendiendo su naturaleza física, tamaño de la perturbación y escala de tiempo, con énfasis en los fenómenos oscilatorios de pequeña señal. Con el fin de mejorar la estabilidad de pequeñas señales, se implementa el ajuste del estabilizador de potencia 2B propuesto por la IEEE, y que no está implementado en la herramienta, utilizando la herramienta Simulink de Matlab versión R2017a, se toman como esquema base el propuesto en Kundur (1993). El comportamiento que presenta el modelo mostrado ante una perturbación es comparado con modelos ya implementados en la herramienta, observándose un mejor comportamiento y confiabilidad.
\end{abstract}

Palabras clave: Estabilizador de Potencia, Estabilidad, Simulink, PSS.

\section{Implementation of the Model Pss2b in Simulink}

\begin{abstract}
In the present work, a study of the different types of stability that are present in Electrical Power Systems (SEP) was carried out, taking into account their physical nature, size of the disturbance and time scale, with emphasis on smallsignal oscillatory phenomena. In order to improve the stability of small signals, the adjustment of the power stabilizer 2B, proposed by the IEEE, which is not implemented in the tool, is implemented using the Simulink tool of Matlab version R2017a, the one proposed in Kundur (1993). The behavior of the model presented before a disturbance is compared with models already implemented in the tool, observing a better behavior and reliability.
\end{abstract}

Keywords: Stabilizer of Power, Stability, Simulink, PSS.

\section{INTRODUCCIÓN}

Como afirman Kundur (1993) y Eufracio (2016) en la actualidad la demanda de energía tiene un crecimiento exponencial, y debido a la necesidad de operar y de producir energía en los sistemas eléctricos de potencias (SEP) se ha incrementado el uso de la generación distribuida, principalmente de las energías renovables, teniendo como resultado que los sistemas eléctricos sean en la actualidad más mallados manejándose grandes bloques de energía por lo que necesario los estudios de estabilidad de voltaje.

En correspondencia con López (2004), los SEP muy cargados son propensos a problemas de la estabilidad de voltaje. Las causas de aparición del colapso de voltaje pueden ser originadas por varias razones, dentro de las cuales se encuentran principalmente: los ajustes de los sistemas de transmisión flexible en corriente alternan (FACTS), así como los sistemas de control de los generadores; régimen de operación del SEP; los límites de la generación para controlar el voltaje y la potencia reactiva y las características de la carga.
Uno de los fenómenos que más afecta a la estabilidad de los SEP, es la estabilidad ante pequeñas oscilaciones, la cual tiene un rango entre 0,1 a 3,0 Hz. Se plantea que estas oscilaciones se relacionan con los ajustes de los sistemas de control de los generadores, con interacciones entre estos o con la acción de la regulación secundaria de frecuencia.

Estas oscilaciones se pueden clasificar de acuerdo a su naturaleza física en oscilaciones mecánicas, electromecánicas locales e inter - área, torsionales y oscilaciones de control.

Para realizar los estudios de estabilidad mediante la simulación hay que tener en cuenta diferentes perturbaciones como son las variaciones en la carga, cortocircuitos que provoquen cambios en la transferencia de potencia de las líneas, cambios de velocidad en los rotores de los generadores, variación de los niveles de voltaje, cambios en la configuración del SEP o en sus parámetros de generación. 
Para mejorar la estabilidad de pequeña señal, se instalan en el SEP los estabilizadores de potencias (PSS) donde su objetivo es mejorar el amortiguamiento de oscilaciones electromecánicas para determinadas frecuencias en uno o más puntos de operación del sistema (Kundur, 1993; GonzalezLongatt, 2006), por lo que el objetivo del presente trabajo es implementar el estabilizador de potencia $2 \mathrm{~B}$, por sus siglas en inglés PSS2B, propuesto por la IEEE en el Simulink de Matlab, tomando, para su validación como esquema base el planteado en Kundur (1993).

Dada la importancia y la necesidad que se tiene de conocer el comportamiento de los equipamientos estabilizadores de potencia ante problemas de estabilidad surge este trabajo en el cual se revisaron diferentes bibliografías con el objetivo de asimilar los conocimientos y conceptos sobre estabilidad ante pequeñas oscilaciones, también se implementó el PSS-2B, propuesto por la IEEE, en la herramienta de Simulink, utilizándose como caso de estudio el sistema de dos aéreas presentado por Kundur (1993).

\section{FUNDAMENTACIÓN TEÓRICA}

Teniendo en cuenta a Kundur (1993) y Gonzalez-Longatt (2006) la estabilidad de un SEP es la propiedad de recuperar el estado de equilibrio en operación después de ocurrida una perturbación.

Ante una perturbación el SEP responde principalmente con variaciones en los ángulos de las máquinas generadoras, en los módulos de los voltajes de los nodos generadores y/o más cargados y oscilaciones en los flujos de potencia. Si la variación angular entre los generadores se mantiene entre los límites permisibles para el SEP, este mantiene el sincronismo, de lo contrario pierde el sincronismo, en la literatura consultada se plantea que este fenómeno se pone de manifiesto transcurridos 2 o 3 segundos desde la perturbación.

La estabilidad de un SEP puede ser clasificada como afirman Gonzalez-Longatt (2006) y Zea (2012) de acuerdo a su naturaleza física, tamaño de la perturbación y escala de tiempo. En la Figura 1 se muestra un resumen de la clasificación del problema de estabilidad en los SEP.

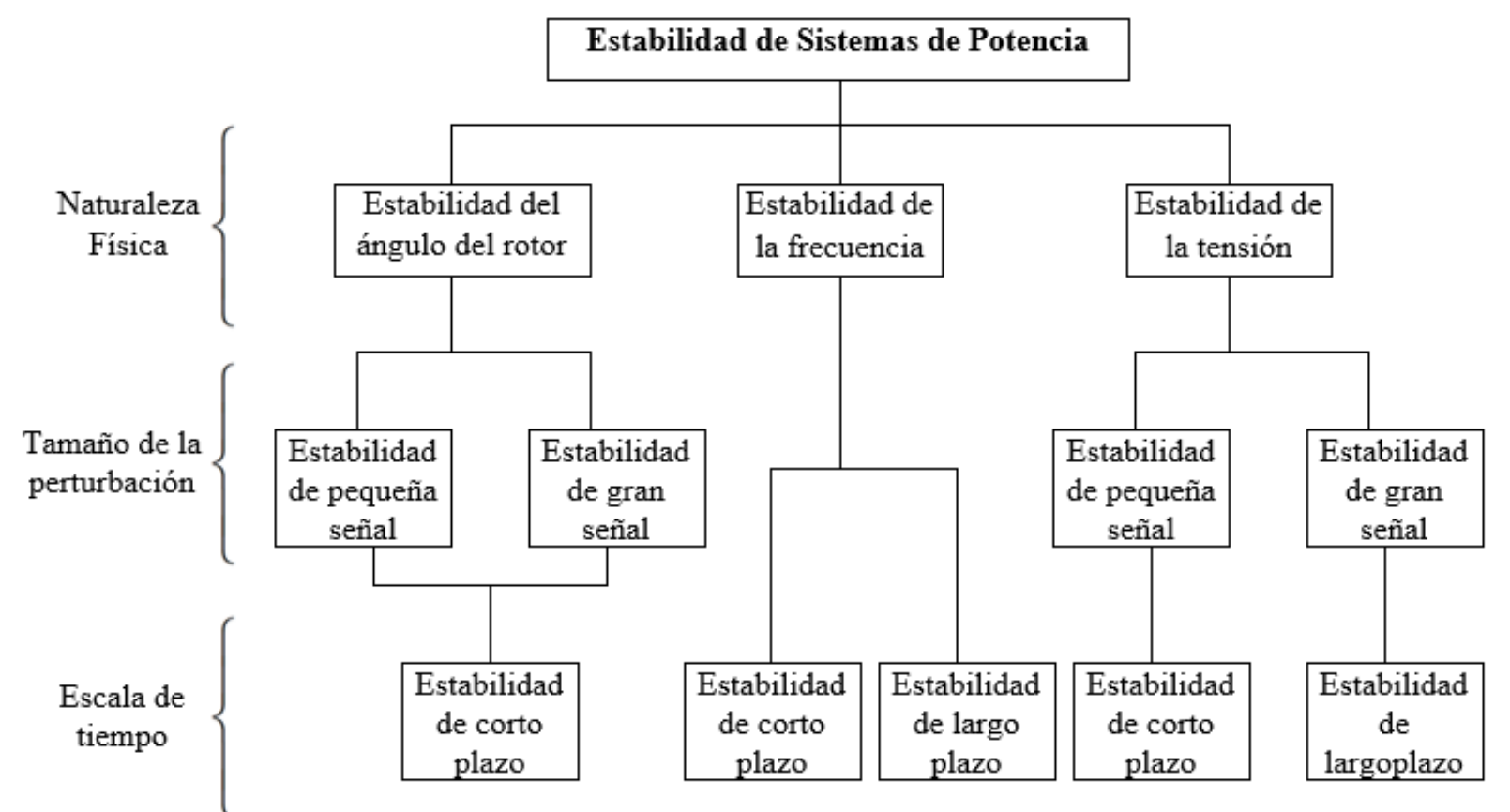

Figura 1. Clasificación de Estabilidad en Sistemas Eléctricos de Potencia. (Fuente: (Zea, 2012))

\subsection{Estabilidad de pequeña señal}

La estabilidad ante pequeñas perturbaciones o pequeña señal se define, teniendo en cuenta a Kundur (1993) y Zea (2012), como la propiedad de los generadores sincrónicos de mantenerse en sincronismo ante una pequeña perturbación y depende de la capacidad de mantener o recuperar el estado de equilibrio entre los torques electromagnéticos y los torques mecánicos de cada generador del SEP, en este contexto se consideran pequeñas perturbaciones a las pequeñas variaciones que ocurren en la carga y/o en la generación

La respuesta del SEP ante pequeñas señales, afirman Gonzalez-Longatt (2006) e Idárraga (2008), depende principalmente del estado inicial de operación, de la fortaleza del sistema de transmisión, y las características del control de excitación de los generadores.

La estabilidad ante pequeñas señales se debe principalmente a la falta de amortiguamiento en las oscilaciones, las cuales pueden surgir debido a cambios en la topología y/o el punto de operación del SEP, estas pueden ser pequeñas, como ya se mencionó, como cambios constantes en la carga o grandes como una falla trifásica en una línea de trasmisión por la cual circule una gran cantidad de potencia.

En los SEP, en correspondencia con Kundur (1993) las oscilaciones pueden clasificarse como: 
- Oscilaciones normales o positivamente amortiguadas. Ocurren debido a eventos de rutina en los SEP, así, por ejemplo: para cambios de carga, salida de generadores y maniobras que pueden causar oscilaciones en el flujo de potencia, voltaje, corriente $\mathrm{y}$ frecuencia, el sistema no tiene problemas para reducir la amplitud de este tipo de oscilaciones.

- Oscilaciones sostenidas o no amortiguadas. Este tipo de oscilaciones se auto sustentan y no desaparecen sin una acción correctiva. Las oscilaciones sostenidas no son crecientes, pero tampoco tienden a reducirse. Este tipo de oscilaciones son dañinas en el sistema si tienen una amplitud suficientemente grande.

- Oscilaciones amortiguadas negativamente. Si una oscilación aparece y crece gradualmente en magnitud, es amortiguada negativamente. Este tipo de oscilación puede aparecer como oscilación normal o sostenida y crecer en tamaño hasta alcanzar una amplitud que los SEP no pueden asimilar por mucho tiempo.

Las oscilaciones debidas a las pequeñas señales, son ocasionadas constantemente, debido a los cambios de los ajustes en la generación, las variaciones de demanda, en los dispositivos de control, etc. Estas son de baja frecuencia y se pueden dividir en los siguientes tipos:

- Oscilaciones electromecánicas.

- Oscilaciones asociadas con los dispositivos de controles.

- Oscilaciones subsincrónicas.

En SEP con un gran número de nodos, estas oscilaciones tienen naturaleza local o global, las cuales están asociadas a modos de oscilación del SEP, pudiendo definirse de acuerdo con Kundur (1993), Idárraga (2008) y Ardanuy (2003) como:

- Modos locales o modos de máquina-sistema: El término local es usado porque se deben a oscilaciones entre generadores de la misma planta o de plantas muy cercanas, o de un generador o una planta contra el resto del SEP. Pueden deberse también a los ajustes incorrectos en los modos de control, por ejemplo, los reguladores automáticos de voltaje de sistemas de excitación (AVR, por sus siglas en inglés) de grandes generadores y que están conectados al SEP a través de interconexiones débiles, líneas muy largas y radiales. El amortiguamiento de estas oscilaciones se logra con la conexión adicional al control de excitación de estabilizadores de potencia (PSS). Las frecuencias de estos modos de oscilación están en el rango de 0,7 a 2,0 Hz.

- Los modos inter-áreas: son las oscilaciones que ocurren entre un grupo de generadores de un área del SEP contra otro grupo de generadores de otra área cercana del SEP interconectadas por líneas cercanas a su capacidad estática de transmisión, es decir, conexiones débiles. Estos modos de oscilación se pueden presentar de dos maneras:

$>$ A frecuencias bajas involucrando todas máquinas generadoras del SEP, dividiendo a este en dos áreas, máquinas de un aérea oscilando contra máquinas de la otra área. Las frecuencias de estos modos de oscilación están en el rango de 0,1 a $0,3 \mathrm{~Hz}$.

$>$ A frecuencias altas involucrando a subgrupos de máquinas generadoras del SEP, oscilando unas contra otras. Las frecuencias de estos modos de oscilación están en el rango de 0,4 a $0,7 \mathrm{~Hz}$.

Se concluye entonces que la frecuencia de oscilación para este modo es de 0,1 a $0,7 \mathrm{~Hz}$.

De acuerdo con Ardanuy (2003), la importancia de los conocimientos de la estabilidad de pequeñas señales, se debe a que:

- Son oscilaciones que afectan a todo SEP.

- Son difíciles de amortiguar (especialmente los interáreas).

Se debe aclarar que el término "estudio de estabilidad de pequeña señal" es utilizado de acuerdo con la literatura especializada que fue consultada, solo para referirse a la estabilidad de los modos locales e inter - áreas, los cuales involucran la estabilidad de las oscilaciones electromecánicas $\mathrm{u}$ oscilaciones de potencia.

López (2004) afirma que, para SEP cargados, con interconexiones a través de líneas de trasmisión débiles y que sea evidente una estructura longitudinal, son expuestos a presentar oscilaciones de baja frecuencia.

Por su parte Idárraga (2008) y Martínez et al. (2015) plantean que una solución factible a este problema, son los PSS, los cuáles pueden ser ajustados, los modos locales, a través de un modelo preciso de una pequeña parte del SEP y simplificar el resto del sistema mediante modelos equivalentes. Mientras que para el estudio de los modos inter - áreas, por su complejidad, es necesario una representación detallada de todo el SEP.

\subsection{El PSS}

Como señala Gallardo (2009) para un adecuado estudio de la interconexión de los SEP es necesario analizar las oscilaciones electromecánicas, pues estás generalmente aumentan cuando se interconectan grandes SEP, mediante enlaces débiles y que además se intercambian grandes bloques de potencia, lo cual puede ocasionar la inestabilidad del sistema.

Para el buen funcionamiento del SEP todos los generadores deben mantener el sincronismo para garantizar de esta forma el valor constante de la frecuencia del voltaje de salida. El AVR es el dispositivo encargado de mantener constante el voltaje y la frecuencia generadas.

En estudios de interconexiones realizados han ocurrido inestabilidades en el sistema, observándose oscilaciones de baja frecuencia en el rango entre 0,1 a $3 \mathrm{~Hz}$, las cuales restringen la capacidad de transmisión de potencia entre las máquinas generadoras y las cargas, es por esta razón que se diseñan los PSS, el cual es un elemento de control adicional sistema de excitación del generador, como se muestra en la 
Figura 2, que permite amortiguar las oscilaciones y estabilizar los SEP. Por lo que, como afirman y Martínez et al. (2015) es necesario para amortiguar estas oscilaciones un buen ajuste del PSS.

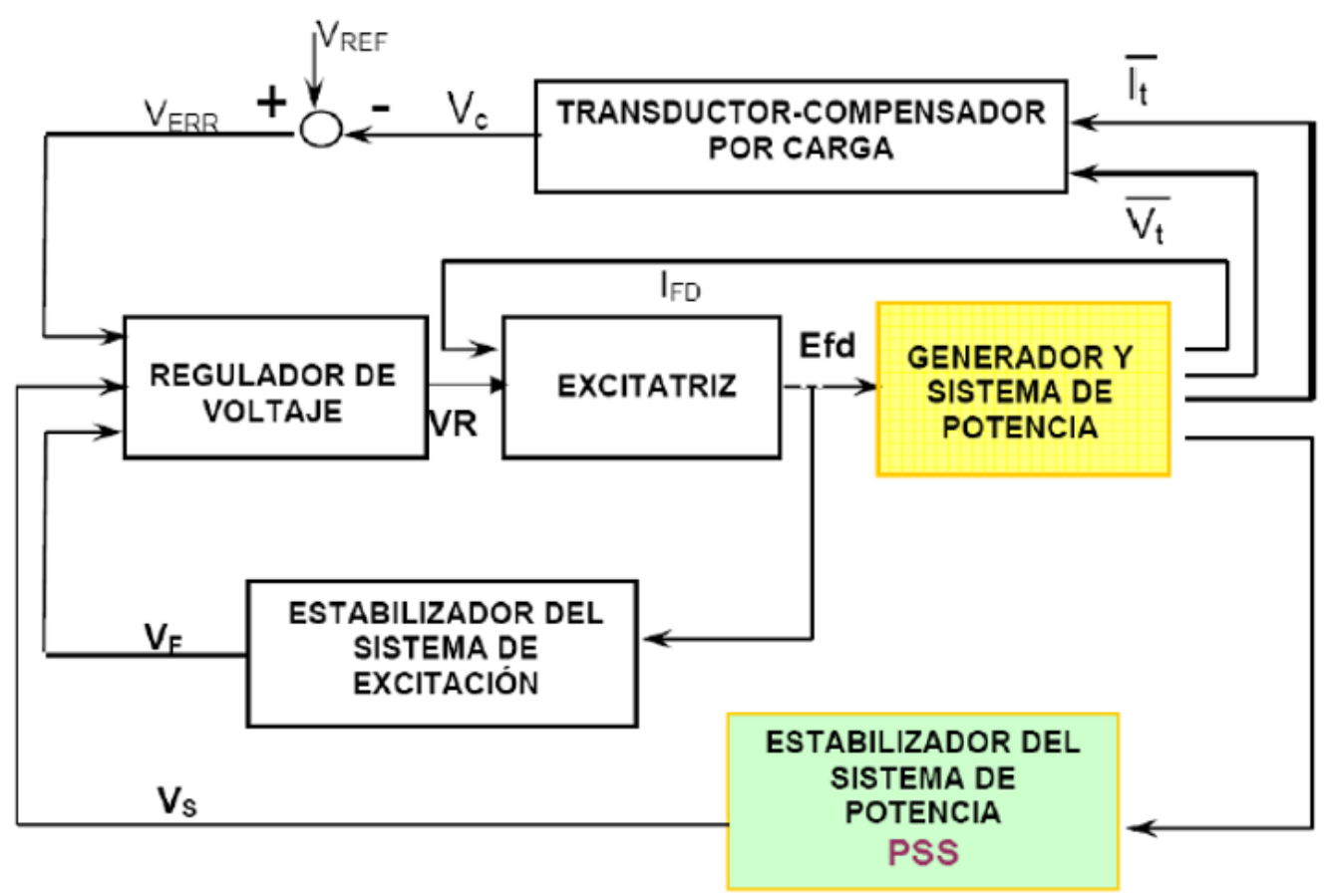

a)

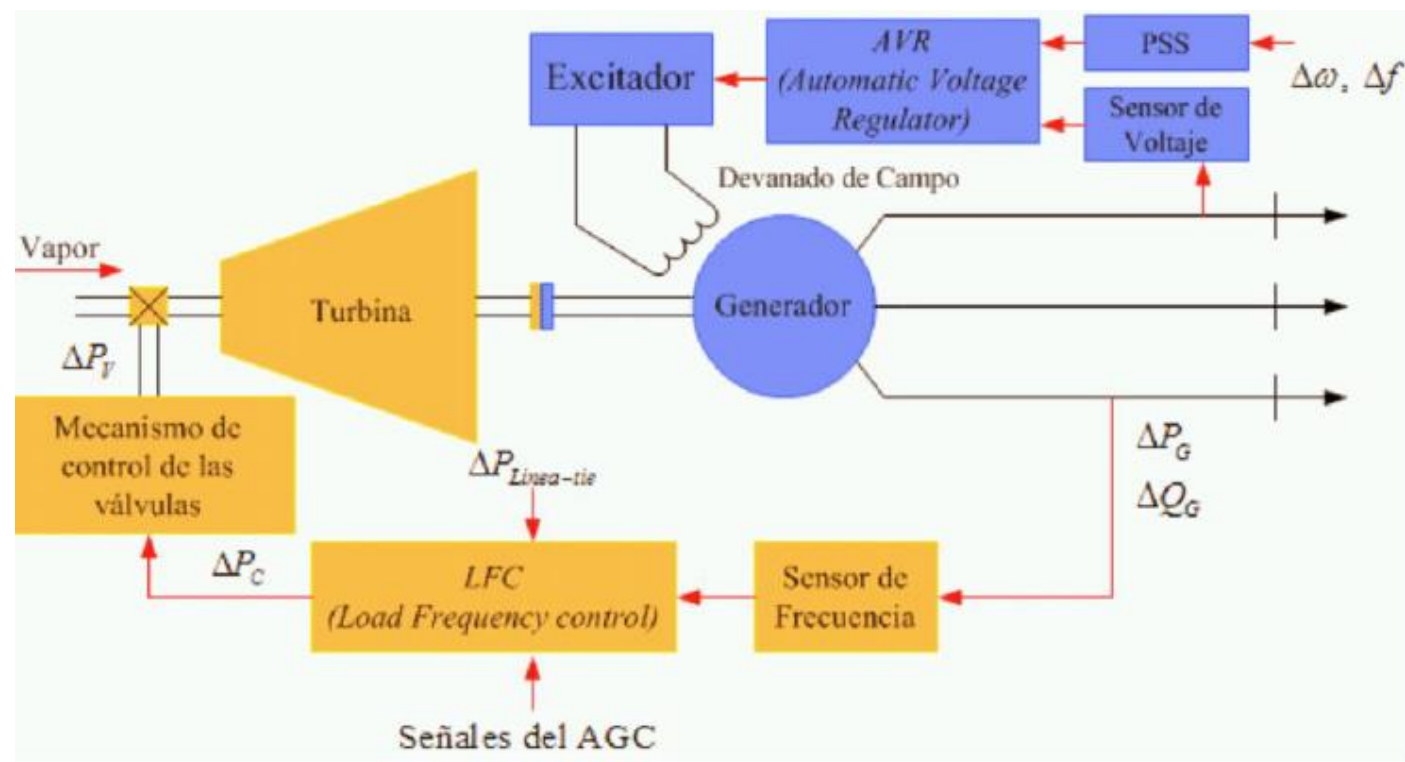

b)

Figura 2. Esquema a) Estabilizador de potencia. b) Esquema de AVR más PSS. (Fuente: (Sánchez, Pérez, \& Castrillon, 2012))

Los PSS son dispositivos ampliamente usados para el amortiguamiento, no solo de las oscilaciones naturales, llamadas modo local de unidades de generación, sino de oscilaciones inter-planta e inter-área. Su objetivo es inyectar una señal adicional al regulador de voltaje (AVR) en fase con las desviaciones de velocidad y de esta forma generar un momento eléctrico amortiguante.
Sánchez et al. (2012) afirma que para que el PSS pueda producir esta señal, debe medir la desviación de velocidad, además de compensar los retardos existentes en el sistema de excitación y el campo del generador, con ajustes apropiados de las constantes de tiempo de dos o más filtros adelanto - atraso. En la Figura 3, se observa un diagrama general de la estructura de un PSS. 


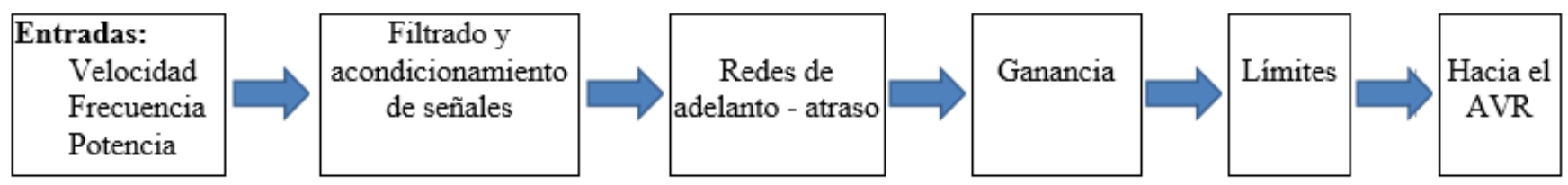

Figura 3. Diagrama general de la estructura de un PSS. (Fuente: (Sánchez, Pérez, \& Castrillon, 2012))

Los PSS se clasifican de acuerdo a la señal de entrada, como: estabilizador delta-omega utiliza la velocidad del ángulo de rotor como señal de entrada; estabilizador delta-f usa la frecuencia eléctrica en la barra de conexión como señal de entrada; estabilizador delta-P usa la potencia como señal de entrada, y estabilizador delta P-omega es una combinación de los tipos a) y c).

Los primeros PSS medían las desviaciones de velocidad en el rotor para generar la señal estabilizante, sin embargo, este tipo de PSS es propenso a incrementar oscilaciones torsionales en unidades térmicas, por lo que se requiere la adición de un filtro torsional que adiciona un desfase a bajas frecuencias, que puede propiciar inestabilidad, lo que impide el uso de altas ganancias y por lo tanto su acción amortiguadora no es la deseada.

La frecuencia eléctrica, como entrada al PSS resulta en un mejor amortiguamiento de los modos de oscilación inter área, ya que esta señal refleja mejor este tipo de oscilaciones. Este tipo de PSS resulta ideal para unidades conectadas a sistemas débiles. No obstante, no son muy sensibles para amortiguar modos locales. Adicionalmente, siguen requiriendo del uso de filtros torsionales en unidades térmicas, además de trasladar al voltaje del generador cualquier ruido en la señal de frecuencia causada por ejemplo por cargas industriales o eventos transitorios en el sistema de potencia. Para eliminar las desventajas de los PSS con entrada de velocidad o frecuencia, se creó el PSS de potencia acelerante, el cual usa como entradas las señales de potencia generada y velocidad o frecuencia, para producir una señal proporcional a las variaciones de velocidad en el rotor, derivada de la potencia acelerante.

De acuerdo con Martínez et al. (2015), para ubicar en que generador o generadores debe estar el PSS se debe partir de un flujo de cargas del SEP en estudio para condiciones normales de operación, tomando este como régimen inicial, luego se incrementarán los voltajes a la entrada de los AVR en un $5 \%$ para realizar corridas de flujo en el dominio del tiempo con el objetivo de observar las variaciones de velocidad en todos los generadores del SEP, siendo la mejor ubicación del PSS en o los generadores que se produzcan las mayores variaciones.

\section{ANÁLISIS Y DICUSIÓN DE LOS RESULTADOS}

Para la implementación del PSS 2B se tomó como caso estudio el sistema de dos aéreas presentado por Kundur (1993), mostrado en la Figura 4.

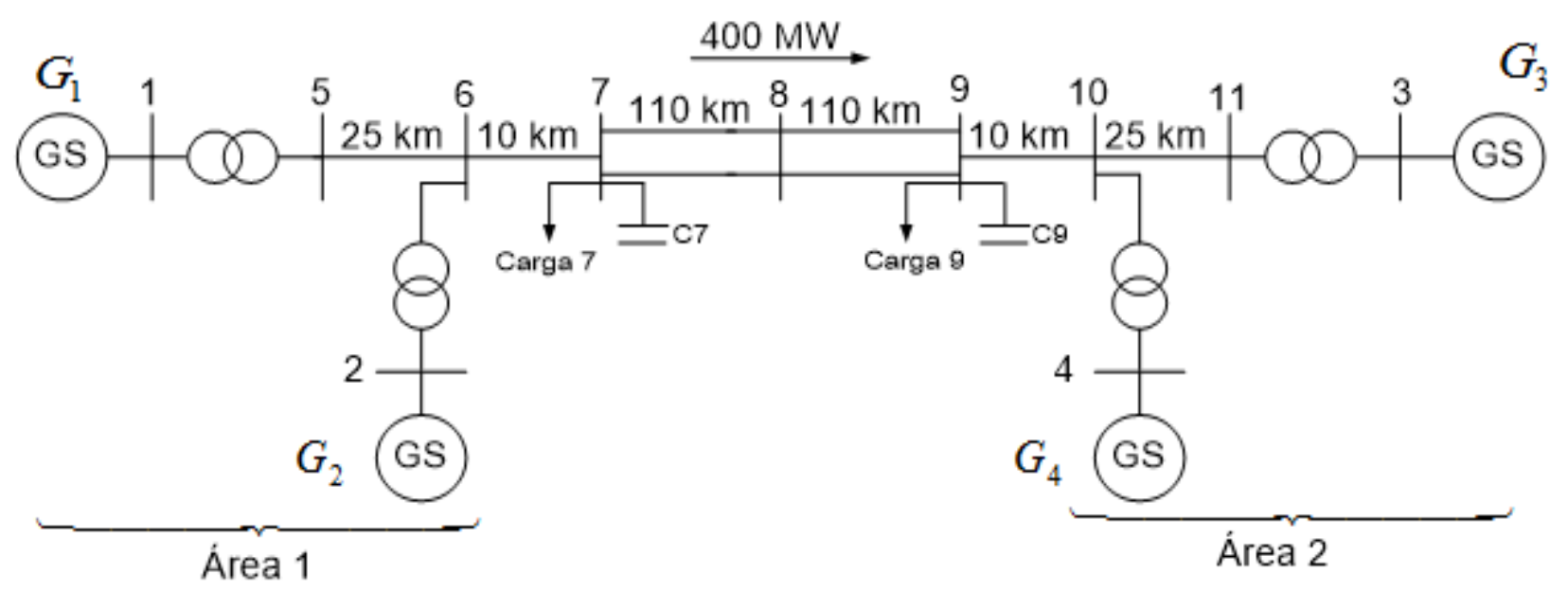

Figura 4. Sistema tomado como base. (Fuente: (Kundur P., 1993))

Este sistema está implementado en la herramienta

SIMULINK/ MATLAB como se muestra en la Figura 5. 


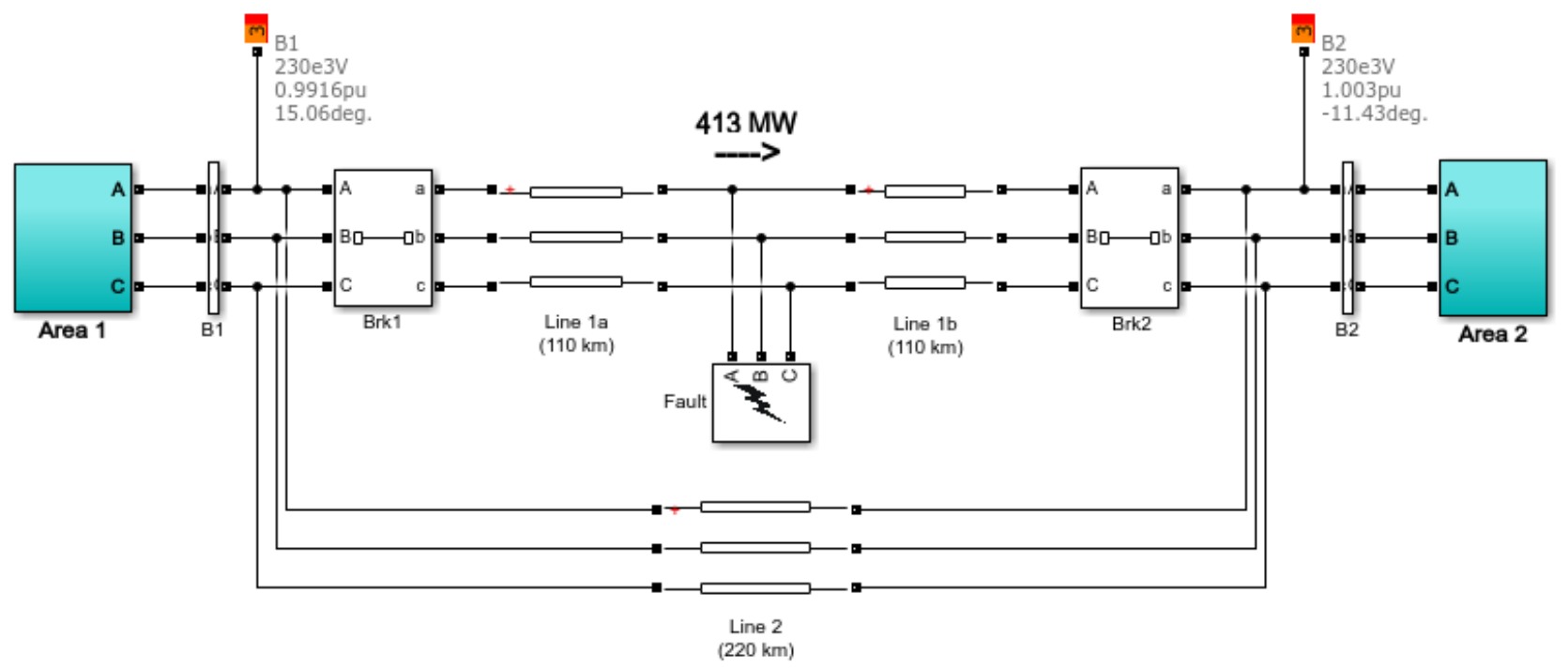

Figura 5. Modelo implementado en Simulink

Cada área está equipada con dos generadores idénticos de 20 kV / 900 MVA, como se aprecia en la Figura 6.

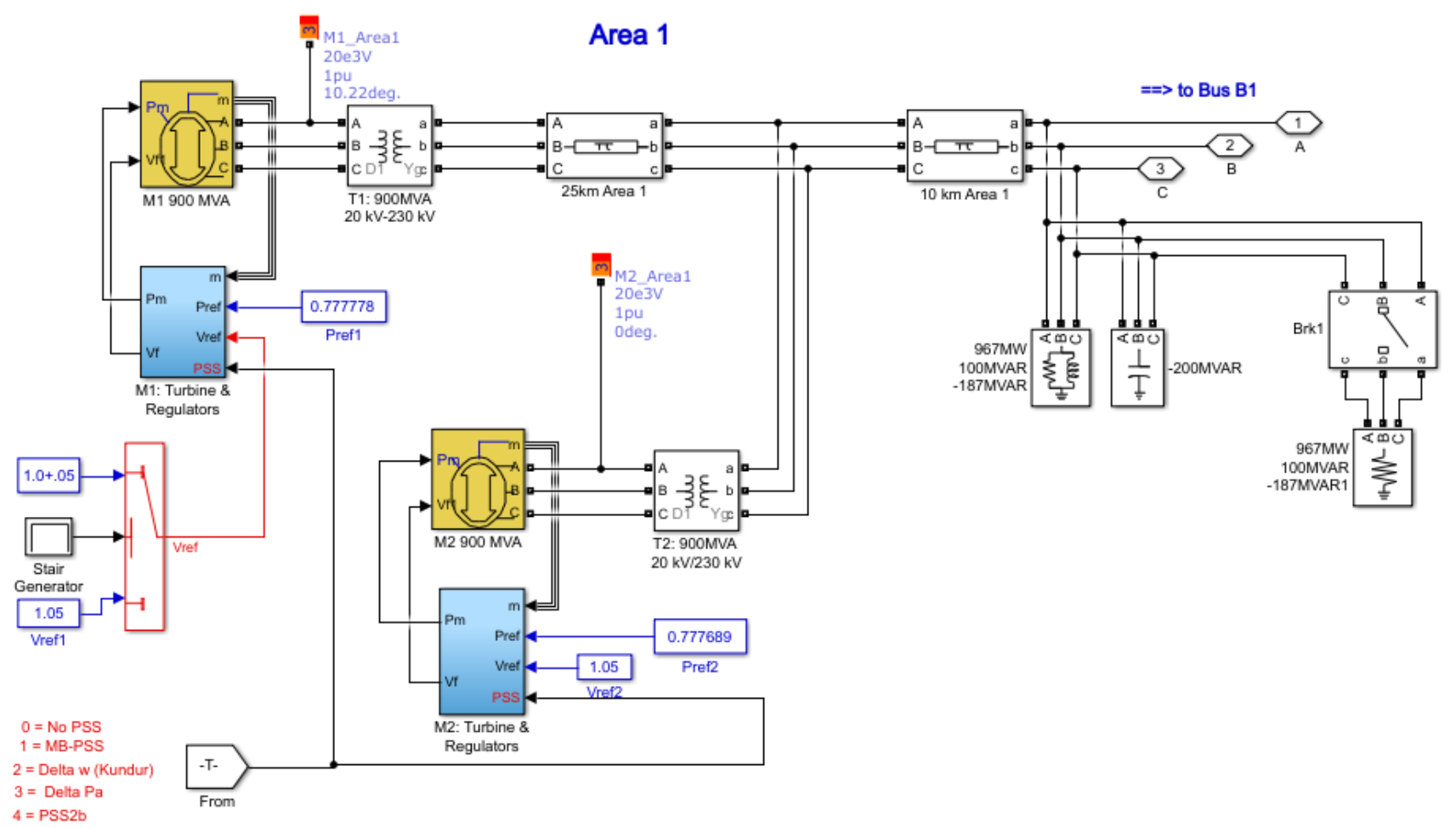

a) 


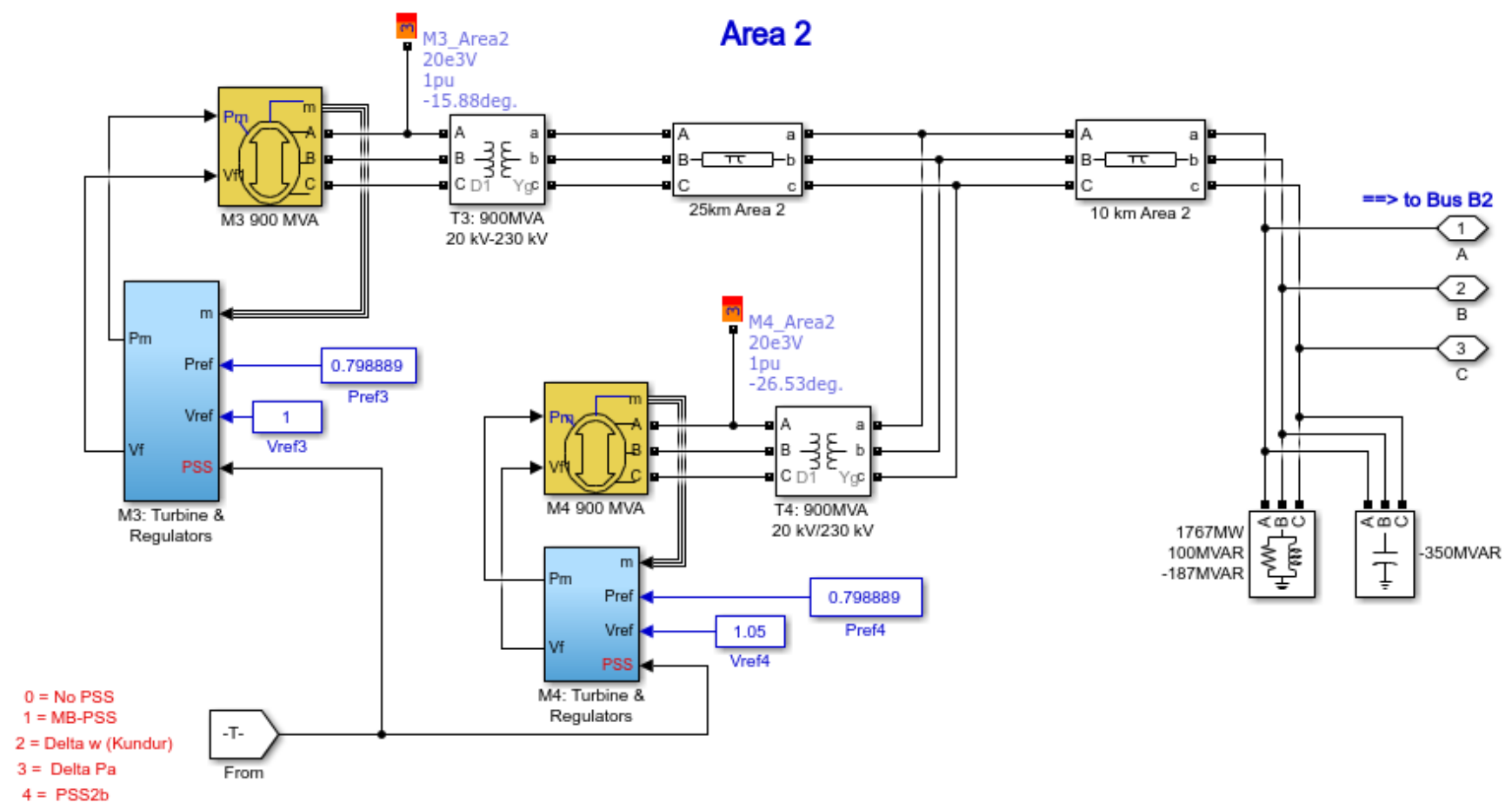

b)

Figura 6. Modelo implementado en Simulink a) área 1 b) área 2

\subsection{Descripción del sistema base}

El sistema de prueba presenta dos áreas totalmente simétricas unidas entre sí por dos líneas de trasmisión de 230 kV de 220 km de longitud. Fue diseñado en Kundur (1993) para realizar estudios de pequeñas señales en SEP grandes. A pesar de su pequeño tamaño, imita muy de cerca el comportamiento de los sistemas típicos en funcionamiento real. Cada área está equipada con dos generadores idénticos de $20 \mathrm{kV} / 900$ MVA, las máquinas síncronas tienen parámetros idénticos, a excepción de las inercias que son $\mathrm{H}=6,5$ en el área 1 y $\mathrm{H}=$ 6,175 en el área 2 . También se suponen plantas térmicas con reguladores de velocidad idénticos en todas las ubicaciones, además de excitadores estáticos rápidos con una ganancia de 200. La carga se representa como impedancias constantes y se divide entre las áreas de tal manera que el área 1 está exportando $413 \mathrm{MW}$ al área 2. Bajo estas condiciones el sistema está al límite de su estabilidad estática. El flujo de carga toma como nodo de balance (de referencia) la máquina 2 y se considera que todos los generadores producen alrededor de 700 MW cada uno. Además, las pérdidas de transmisión y generación pueden variar dependiendo del nivel de detalle con el cual se incorporen los datos de los elementos presentes en el SEP. En el esquema también está presente el bloque Fault, con el cual se puede establecer el tiempo, duración y tipo de cortocircuito.

En el esquema de Simulink, están implementadas para cada generador tres variantes PSS:

- MB-PSS o estabilizador de potencia multibanda: a diferencia de los estabilizadores de potencia o frecuencia convencionales, que tienen un solo filtro de retraso de avance para manejar todos los fenómenos oscilatorios, el MB PSS tiene tres bandas de trabajo ajustables que proporcionan ajustes adecuados en todo el rango de frecuencias. Además de su diseño multibanda, el estabilizador el cual utiliza como señal de entrada la velocidad del rotor, que deriva de las lecturas de voltaje y corriente del terminal del generador.

- Delta $\omega$ PSS o estabilizador de potencia $\Delta \omega$ : éste basa su funcionamiento en la constante medición de las variaciones de velocidad de los generadores, estas son enviadas al PSS, que entrega una señal de control al AVR. Esto se implementa mediante la inclusión de dispositivos de medición de la velocidad de rotación de las máquinas de generación y cuyos valores son luego enviados al PSS para ser procesados.

- Delta Pa PSS, su variable de medición es la potencia acelerante o diferencia de potencias entre la potencia mecánica y la potencia eléctrica del sistema de potencia.

Lo que se propone es implementar en el SEP tomado como caso estudio mostrado en Simulink, el modelo de PSS2B que toma como variables de control la potencia acelerante y la variación en la velocidad de la máquina, datos que son procesados por el PSS para enviar una señal de mando al AVR comprobándose el comportamiento del sistema ante contingencias y sus respuestas a los diferentes modos de oscilación, la Figura 7 muestra el diagrama de bloques del PSS IEEE 2B. 


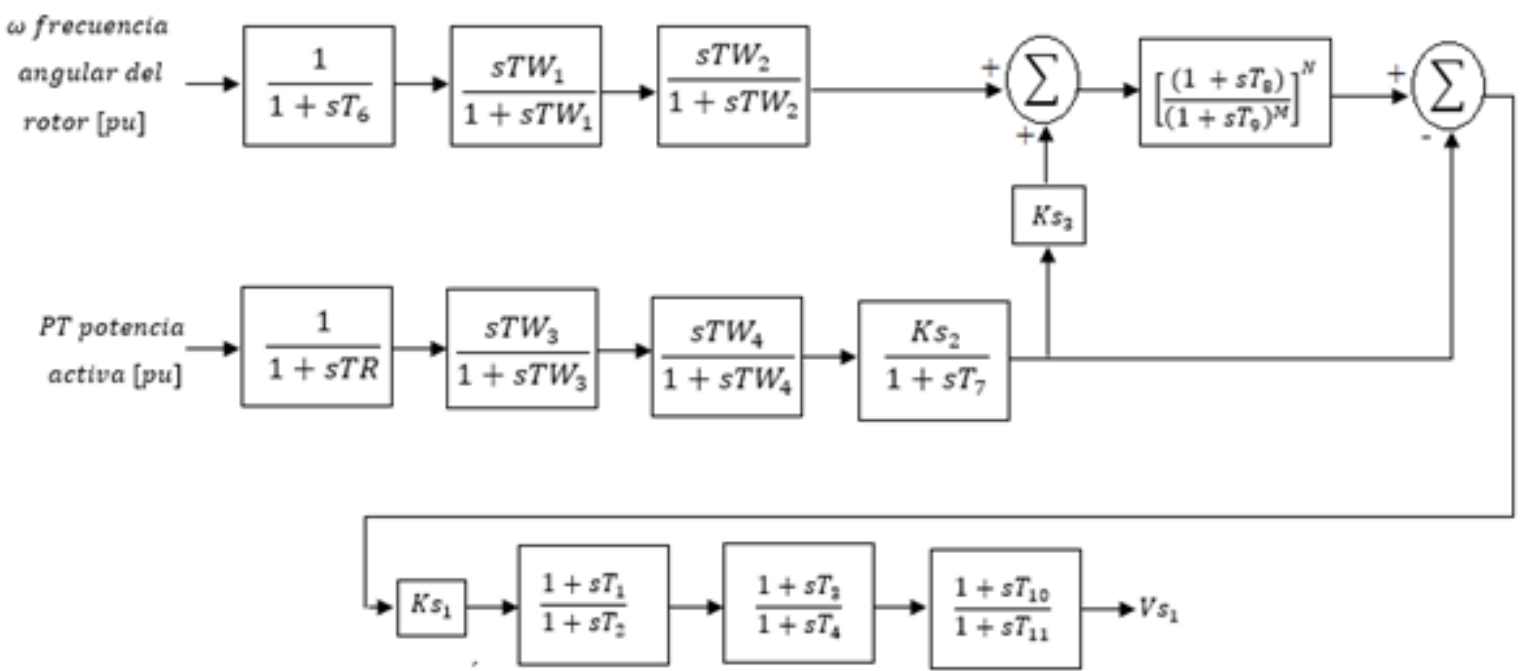

Figura 7. Diagrama de bloques del PSS2B según IEEE

Como afirma Sánchez et al. (2012) la entrada $\omega$ corresponde a la señal de desviación de frecuencia o velocidad, mientras la entrada $P T$ está asociada a la señal de potencia activa generada. Las constantes $T W_{1}$ a $T W_{4}$ son filtros pasa alto, con el propósito de habilitar la acción del PSS cuando ocurran oscilaciones que deban ser amortiguadas, pueden ajustarse entre 1 y 20 segundos. La señal de potencia $P T$ pasa a través de los filtros pasa alto $\left(T W_{3}\right.$ y $\left.T W_{4}\right)$ para ser integrada, y producir la integral de la potencia eléctrica.

En Sánchez et al. (2012) citando a Bérubé \& Hajagos (2007) se sugieren los siguientes criterios de ajuste para las funciones de transferencia de la potencia y frecuencia:

$T W_{3}=T_{7} ; T W_{4}=0 ; K s_{2}=\frac{T W_{1}}{2 H} ; K s_{3}=1$.

Donde $2 \mathrm{H}=\mathrm{M}$ es la inercia del grupo generador - turbina. $T W_{1}=T W_{2}=T W_{3} ; T_{6} \approx 0$.

Las constantes de tiempo $T_{8}$ y $T_{9}$, como los índices M y N, son seleccionados para conformar un filtro pasa bajo, llamado filtro rastreador de rampa, que tiene como objetivo atenuar componentes de alta frecuencia en la señal de entrada, minimizando el efecto en el voltaje del generador cuando se presenten cambios rápidos en la potencia mecánica. En Bérubé \& Hajagos (2007) se presentan los ajustes más usados para el filtro rastreador de rampa: $N=1 ; M=5 ; T 9=0,1 ; T 8=M$. $T 9=0,5$

Las constantes de tiempo $T_{1}, T_{2}, T_{3}$ y $T_{4}$, componen los filtros de adelanto - atraso que son el corazón del PSS. Su función es compensar los retardos generados en el sistema de excitación, los transductores, filtros y el campo del generador. De esta forma, el PSS puede inyectar una señal en fase con las desviaciones de velocidad, y proporcionar el amortiguamiento deseado. Para generar amortiguamiento sobre un amplio rango de frecuencia y reducir los efectos indeseados sobre el voltaje generado, Sánchez et al. (2012) afirman que las constantes de tiempo deben fijarse de tal manera que este retardo sea menor de $90^{\circ}$ para el mayor rango de frecuencia posible, y entre $0^{\circ} \mathrm{y}$ $45^{\circ}$ desde la frecuencia más baja para el modo inter - área hasta la más alta del modo local.

La Figura 8 muestra el PSS2B implementado en Simulik y la Tabla 1 se muestran los valores estándares que se tomaron como ajustes en correspondencia con lo planteado por Martínez et al. (2015) y brindados por Jumbo (2012), DuarteMermoud \& Milla (2018), Verdejo et al. (2015) y Delgado et al. (2017). 


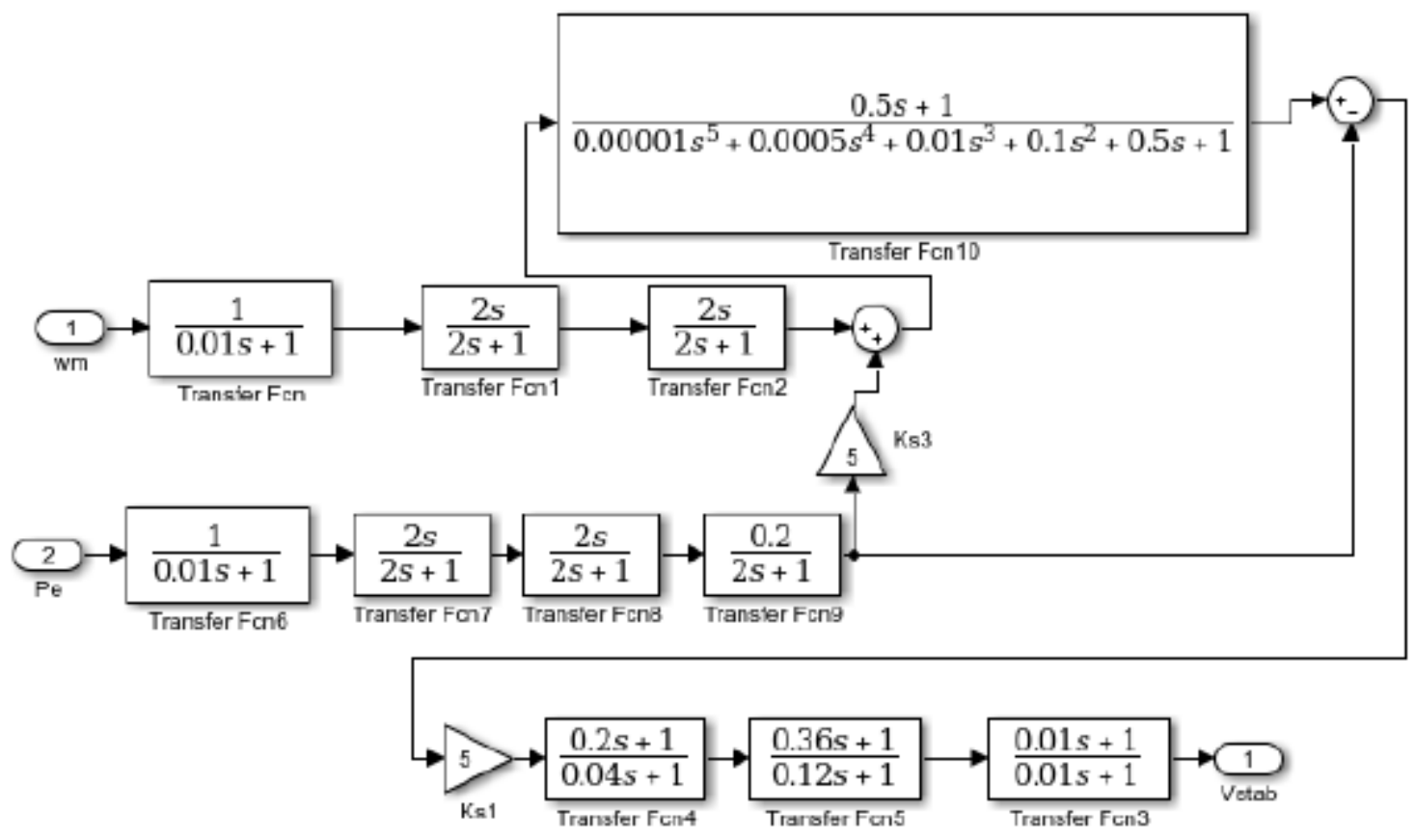

Figura 8. PSS2B implementado en Simulik

\begin{tabular}{|c|c|c|c|}
\hline \multicolumn{4}{|c|}{ Tabla 1. Parámetros de ajuste del PSS2B } \\
\hline Parámetro & Descripción & Unidad & $\begin{array}{c}\text { Valores } \\
\text { estándar }\end{array}$ \\
\hline$T W_{1}, T W_{2}$ & $\begin{array}{l}\text { Constantes de } \\
\text { tiempo de } \\
\text { limpieza. }\end{array}$ & $\mathrm{s}$ & 2,0 \\
\hline$T W_{3}, T W_{4}$ & $\begin{array}{l}\text { Constantes de } \\
\text { tiempo de } \\
\text { limpieza. }\end{array}$ & $\mathrm{s}$ & 2,0 \\
\hline$K s_{1}$ & $\begin{array}{l}\text { Factor de } \\
\text { ganancia del PSS }\end{array}$ & $\mathrm{pu}$ & 5,0 \\
\hline$K s_{2}$ & $\begin{array}{l}\text { Factor de } \\
\text { compensación } \\
\text { para el cálculo } \\
\text { integral de la } \\
\text { potencia eléctrica. }\end{array}$ & $\mathrm{pu}$ & 0,2 \\
\hline$K s_{3}$ & $\begin{array}{l}\text { factor de } \\
\text { coincidencia de } \\
\text { señal. }\end{array}$ & $\mathrm{pu}$ & 1,0 \\
\hline$T_{1}, T_{3}, T_{10}$ & $\begin{array}{l}\text { constantes de } \\
\text { tiempo de entrega } \\
\text { de la red } \\
\text { acondicionada }\end{array}$ & $\mathrm{s}$ & $\begin{array}{c}0,2 \\
0,36 \\
0,01\end{array}$ \\
\hline$T_{2}, T_{4}, T_{11}$ & $\begin{array}{l}\text { constantes de } \\
\text { tiempo de retardo } \\
\text { de la red } \\
\text { acondicionada }\end{array}$ & $\mathrm{s}$ & $\begin{array}{l}0,04 \\
0,12 \\
0,01\end{array}$ \\
\hline$T R$ & $\begin{array}{l}\text { Constante de } \\
\text { tiempo activa del } \\
\text { transductor de } \\
\text { potencia }\end{array}$ & $\mathrm{s}$ & 0,01 \\
\hline
\end{tabular}

Tabla 1. Parámetros de ajuste del PSS2B

\begin{tabular}{|c|c|c|c|}
\hline Parámetro & Descripción & Unidad & $\begin{array}{c}\text { Valores } \\
\text { estándar }\end{array}$ \\
\hline$T_{6}$ & $\begin{array}{l}\text { desviación } \\
\text { angular de } \\
\text { frecuencia del } \\
\text { rotor constante de } \\
\text { tiempo del } \\
\text { transductor }\end{array}$ & S & 0,01 \\
\hline$T_{7}$ & $\begin{array}{l}\text { constante de } \\
\text { tiempo para el } \\
\text { cálculo integral de } \\
\text { la potencia } \\
\text { eléctrica }\end{array}$ & $\mathrm{s}$ & 2,0 \\
\hline$T_{8}$ & $\begin{array}{l}\text { constante de } \\
\text { tiempo de filtro de } \\
\text { seguimiento de } \\
\text { rampa }\end{array}$ & $\mathrm{s}$ & 0,0 \\
\hline$T_{9}$ & $\begin{array}{l}\text { constante de } \\
\text { tiempo de filtro de } \\
\text { seguimiento de } \\
\text { rampa }\end{array}$ & $\mathrm{s}$ & 1,0 \\
\hline M & $\begin{array}{l}\text { rampa de } \\
\text { seguimiento filtro } \\
\text { grado }\end{array}$ & - & 5 \\
\hline $\mathrm{N}$ & $\begin{array}{l}\text { rampa de } \\
\text { seguimiento filtro } \\
\text { grado }\end{array}$ & - & 1 \\
\hline
\end{tabular}




\subsection{Comparación de los resultados.}

En el experimento se programa un cortocircuito trifásico que afecta a la red al segundo uno de la simulación, teniendo una duración de 8 ciclos. Tras la apertura de los interruptores en los extremos de la línea, se elimina el flujo de potencia por dicha línea y se crea un modo oscilatorio. Se partió, como caso 1, de la instalación de los PSSs en las cuatro máquinas con los mismos ajustes, pues así está implementado el ejemplo inicial en el Simulink de Matlab.
Como se puede observar en la Figura 9, el comportamiento del PSS2B con los ajustes realizados el sistema es estable, sobresaliendo ante el $\triangle \mathrm{Pa}$-PSS que demora más en alcanzar un estado estable, más de $20 \mathrm{~s}$, con gran oscilación de la potencia transferida.

Es de recordar que, en toda la literatura, como ya se mencionó anteriormente, no es recomendable la instalación de PSS en todas las máquinas del sistema por lo que se procedió a instalar PSS solo en el generador 4, ejemplo que se tomó como caso 2.

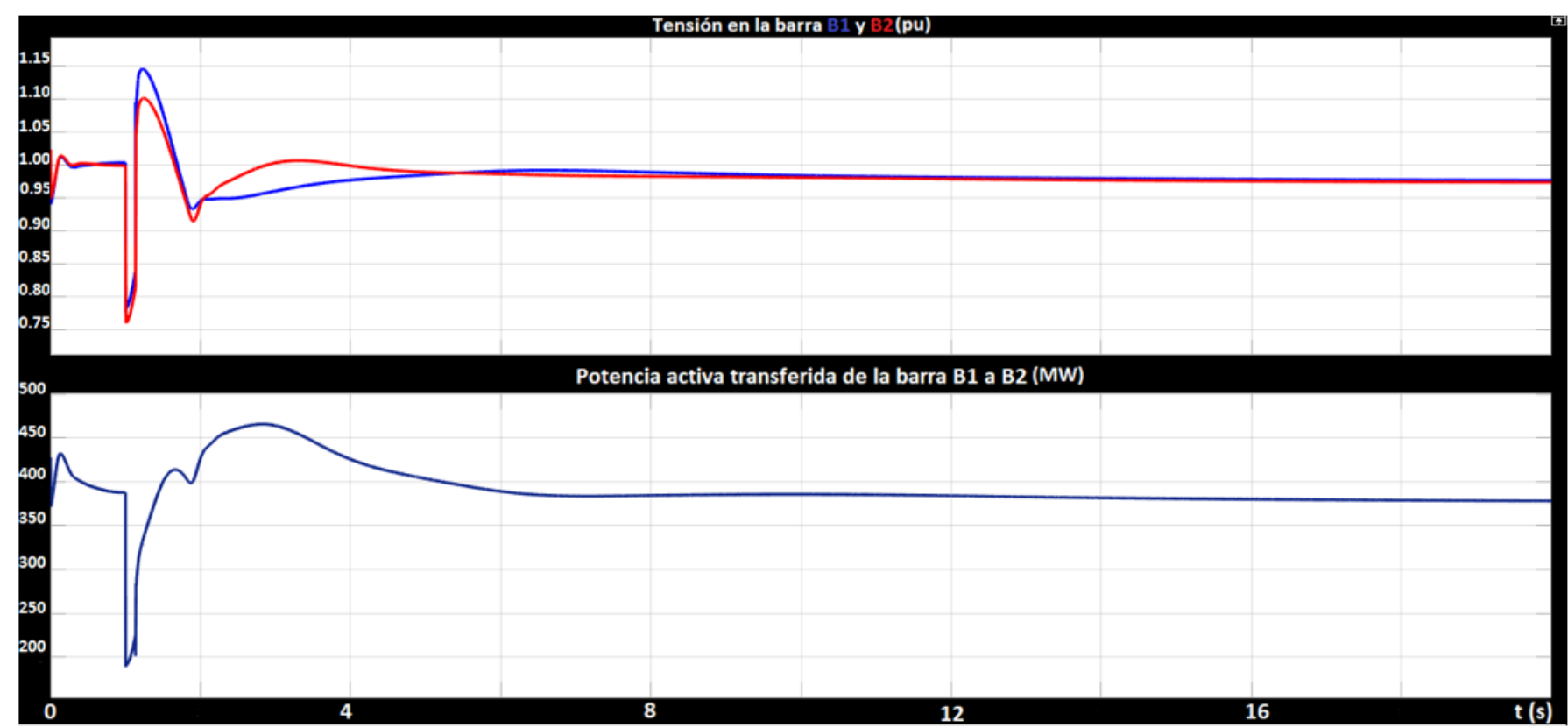

a)

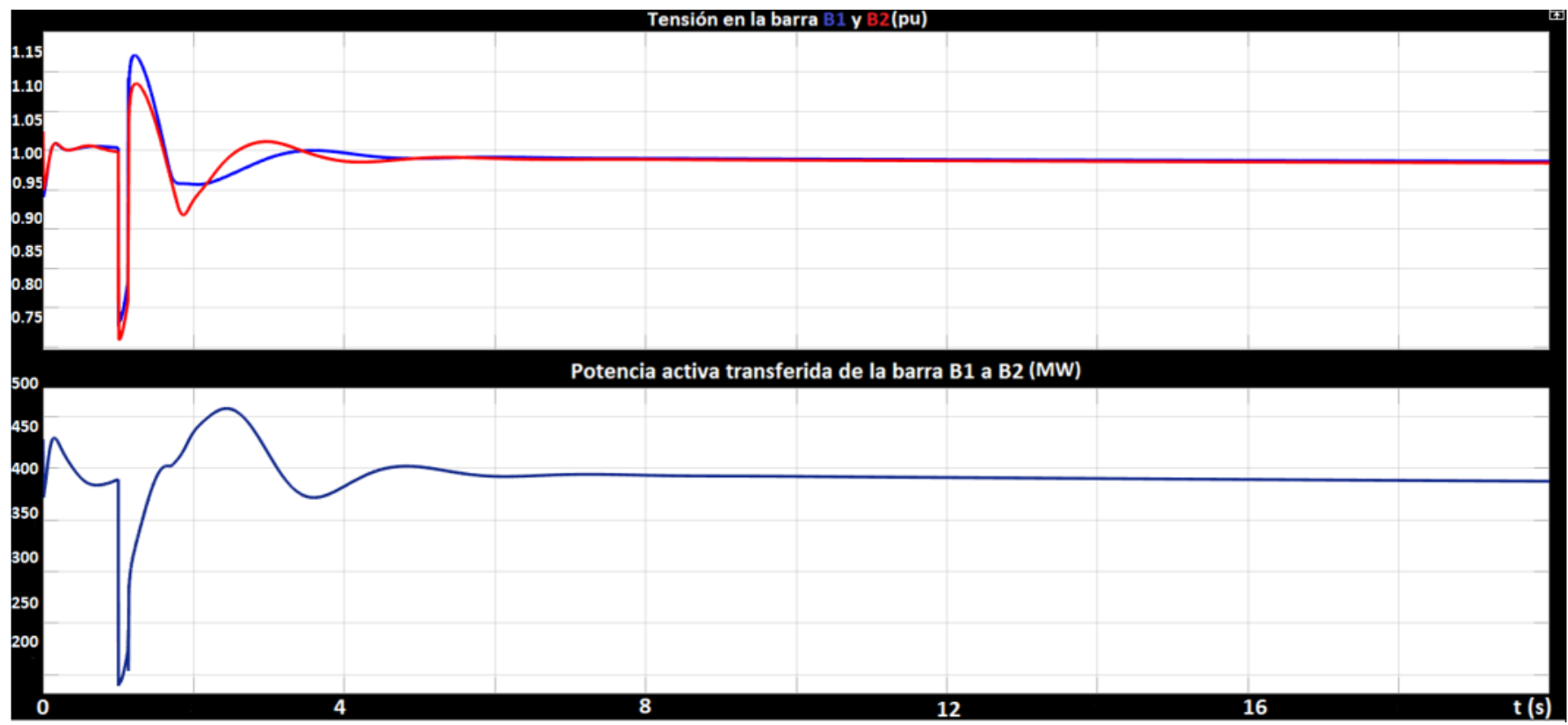



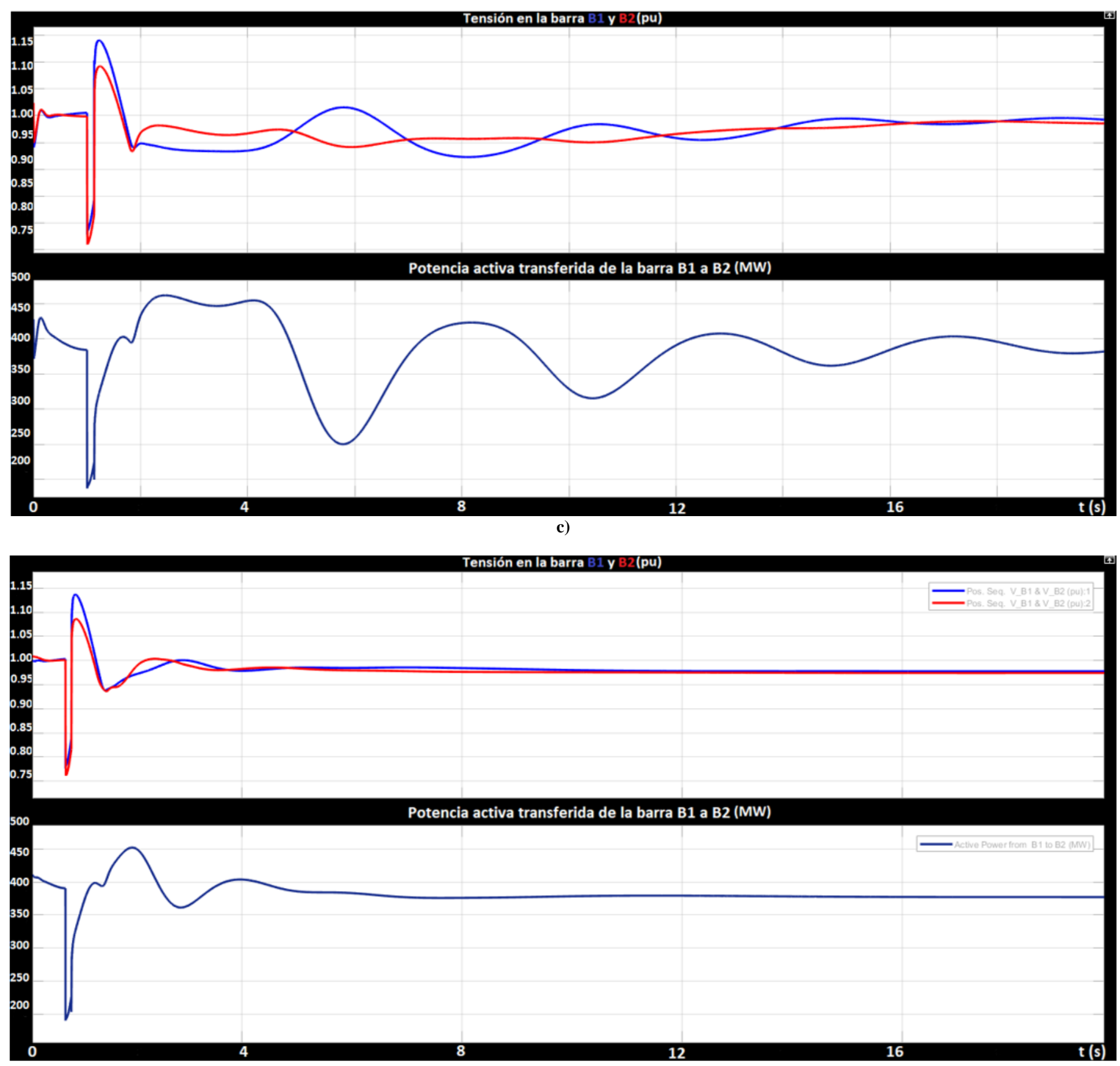

d)

Figura 9. a) respuesta al cortocircuito del MB-PSS, b) respuesta al cortocircuito $\Delta \omega$-PSS, c) respuesta al cortocircuito $\Delta$ Pa-PSS, d) respuesta al cortocircuito PSS2B

Como se puede apreciar en la Figura 10, el MB-PSS no es capaz de amortiguar por sí solo el modo de oscilación que aparece luego del cortocircuito y su posterior aislamiento de la red. Comportamiento similar mostraron el $\triangle \mathrm{Pa}$-PSS y $\Delta \omega$ PSS. El PSS2B por el contrario es capaz de contrarrestar el efecto oscilatorio creado por la contingencia que aparece en el sistema, comportamiento esperado en todo momento. 


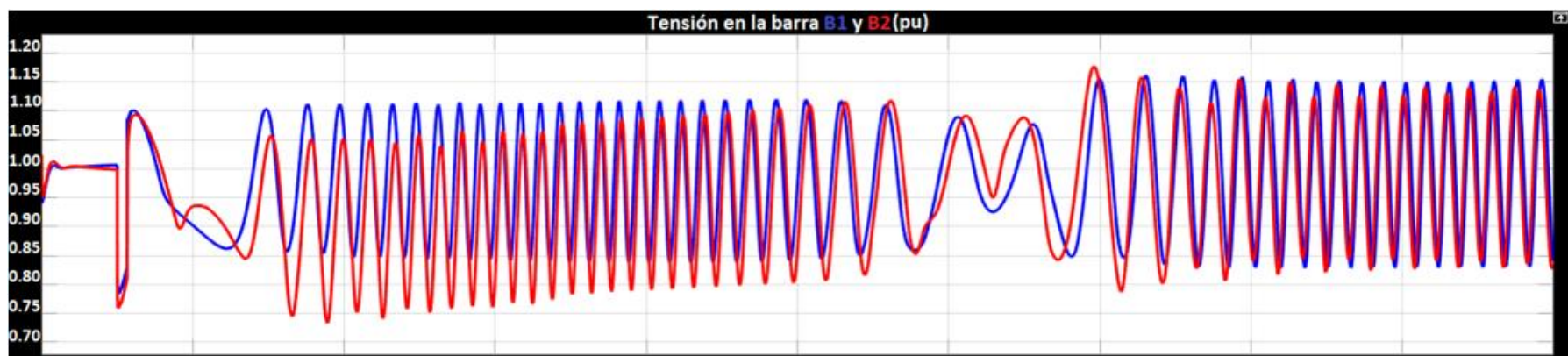

Potencia activa transferida de la barra B1 a B2 (MW)
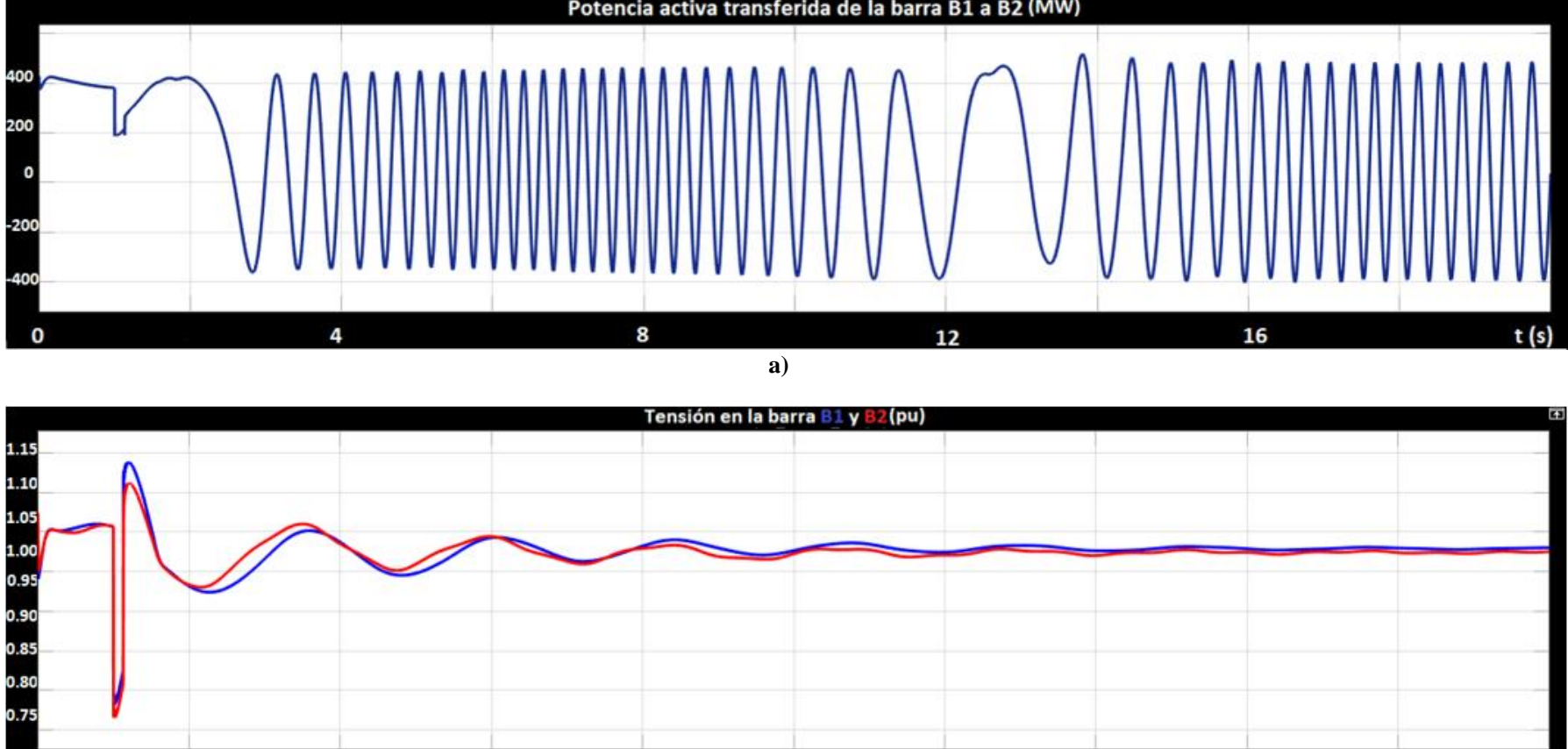

Potencia activa transferida de la barra B1 a B2 (MW)

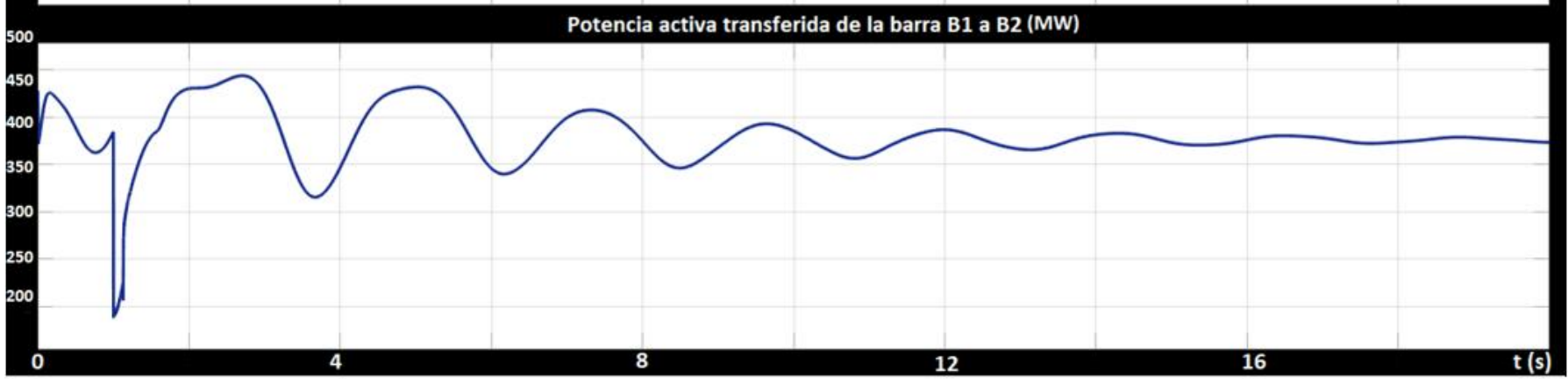

Figura 10. a) resultado de utilizar el MB-PSS solo en la máquina 4, b) Resultado de utilizar el PSS2B de la IEEE solo en la máquina

\section{CONCLUSIONES}

Con la realización de este trabajo se pudo comprobar la importancia de la instalación de PSSs para mejorar la estabilidad ante pequeñas perturbaciones de los SEP, por lo que se formulan las siguientes conclusiones.

- $\quad$ Se implementó el PSS2B de la IEEE usando la herramienta Simscape Power Systems de Matlab y se ajustó en correspondencia a las condiciones necesaria para mantener la estabilidad del sistema tomado como caso de estudio.

- Basándose en el análisis del caso 1, Figura 9, se puede observar que las variaciones de la potencia activa transferida y los voltajes en las barras 1 y 2 del sistema cuando están instalados solamente el PSS MB-PSS y el PSS2B son menores del 5\% (ver
Figura 9 a) y d)), no ocurriendo así con el resto de los PSS instalados, por ejemplo, el $\triangle \mathrm{Pa}$-PSS, empieza a amortiguar las oscilaciones a partir de los $20 \mathrm{~s}$ (ver Figura $9 \mathrm{c}$ )), en cuanto al $\Delta \omega$-PSS, las diferencias de las amplitud de la variación del voltaje durante la contingencia oscilan en el orden del $6,6 \%$ con respecto a los PSS MB-PSS y PSS2B, similar situación sucede con la transferencia de potencia activa siendo del $20 \%$ aproximadamente, por lo que se tomó para el estudio del caso 2 la comparación del MB-PSS y el PSS2B.

- En el caso 2, Figura 10, puede constatarse que con la instalación del MB-PSS, ante la ocurrencia de la contingencia, se pierde la estabilidad del sistema pues las oscilaciones tanto de voltaje 
como de potencia activa transferida no se amortiguan, no sucediendo así con el PSS2B las cuales se amortiguan alrededor de los $12 \mathrm{~s}$, por lo que la instalación de este PSS es la idónea.

\section{REFERENCIAS}

Ardanuy, J. F. (2003). Diseño de un estabilizador de sistemas de potencia neuro-borroso adaptativo ajustado mediante algoritmos genéticos. Tesis doctoral. Universidad Politécnica de Madrid, Escuela Técnica Superior de Ingenieros de Telecomunicaciones,. Recuperado el Diciembre de 2016, de http://oa.upm.es/42/1/09200302.pdf.

Bérubé, G., \& Hajagos, L. (2007). Accelerating-Power Based Power System Stabilizers. Kestrel Power Engineering Ltd. Mississauga, Ontario, Canada. Recuperado el 2017, de https://kestrelpower.com/Docs/PSS_Tutorial_Ch apter_Accelerating_Power_R2.pdf

Casas, L. P. (1991). El estabilizador de potencia en amortiguamiento de las oscilaciones electromecánicas en sistemas eléctricos multimáquinas. $X$ Congreso Nacional de Ingeniería Mecánica y Mecánica Eléctrica. Recuperado el diciembre de 2016, de: http://hrudnick.sitios.ing.uc.cl/paperspdf/estabil.p df.

Delgado, F. R., Velásquez, S., Rosales, L., \& Toledo, J. (2017). Modelado de estabilizador de potencia para análisis de pequeña señal utilizando paquete de simulación. Universidad, Ciencia $Y$ Tecnología. Revista de la universidad nacional experimental politécnica "José Antonio de Sucre". 21(84), 119-125. Recuperado el 2019 de: http://uct.unexpo.edu.ve/index.php/uct/article/do wnload/802/646

Duarte-Mermoud, M. A., \& Milla, F. (2018). Estabilizador de Sistemas de Potencia usando Control Predictivo basado en Modelo. Revista Iberoamericana de Automática e Informática industrial. Universitat Politècnica de València. 17(3), 286-297. doi: 10.4995/riai.2020.13304 Recuperado el 2019, de https://polipapers.upv.es/index.php/RIAI/article/v iew/10056/10191

Eufracio, N. M. (2016). Mejora de la estabilidad transitoria en líneas de gran longitud mediante el uso de FACTS. Tesis maestría. Universidad de Sevilla. Recuperado el marzo de 2018, de http://bibing.us.es/proyectos/abreproy/70786/

Gallardo, Q. C. (2009). Estabilidad y Amortiguamiento de Oscilaciones en Sistemas Eléctricos con Alta Penetración Eólica. Tesis doctoral. Universidad Carlos III de Madrid, Departamento de Ingeniería Eléctrica, Electrónica y Automática. Recuperado el 2017, de: http://orff.uc3m.es/bitstream/handle/10016/6731/ CarlosGallardoTesisdoctoral.pdf?sequence $=1$.

García, A. M., Llanes, M. V., Breffe, O. T., \& Martínez, M. P. (2015). Ajuste de Estabilizadores de Potencia en generadores utilizando el paquete Power
Systems Analysis Toolbox PSAT. XXXVI(1), 8393. La Habana: Ingeniería Energética. Obtenido de http://rie.cujae.edu.cu/index.php/RIE/article/view /424/455

Gonzalez-Longatt, F. M. (2006). Estabilidad en Sistemas de Potencia. Recuperado el diciembre de 2016, de http://fglongatt.org/OLD/Archivos/Archivos/SP_ II/PPTCapitulo2SP2.pdf.

Idárraga, V. M.; Parra, D. (2008). Control de oscilaciones electromecánicas en sistemas eléctricos de potencia usando el análisis modal. Tesis ingeniería. Universidad Tecnológica de Pereira. Recuperado el Diciembre de 2016, de http://repositorio.utp.edu.co/dspace/bitstr

Jumbo, Zhang; Chung,C.-Y.; Yingduo, Han. ( 2012). A novel modal decomposition control and its application to PSS design for damping interarea oscillations in power systems, IEEE Transactions on Power Systems Vol. 27, No. 4. 2015-2025. 10.1109/TPWRS.2012.2188820. Recuperado el 2017 , de https://www.scopus.com/record/display.uri?eid=2 -s2.0-

$84868003093 \&$ origin $=$ inward\&txGid=d22617c6 0c151d1d7c595b48081128f8

Kundur, P. (1993). Power systems stability and control. s.l. : EPRI editors, Neal J. Balu \& Mark G. Lauby.

Kundur, P. e. (mayo de 2004). Definition and Classification of Power System Stability. Recuperado el diciembre de 2017, de https://pdfs.semanticscholar.org/617a/0a813629f 460915909840dc42330ce184718.pdf.

López, P. C.; Fernández, F.; Rauco, L.; Gonzáles, M; Rojo, J.; \& Diogo, J. (2004). Estabilidad de los sistemas eléctricos insulares. . Obtenido de https://www.icai.es/contenidos/publicaciones/ana les_get.php?id=259

Martínez , G., Vilaragut , L., Torres , B., \& Pérez , M. (2015). Ajustes de estabilizadores de potencia en generadores utilizando el paquete Power Systems Analysis Toolbox PSAT, Ingeniería Energética Vol. XXXVI, No. 1.

Resquena-Pérez, R. (2013). Diseño de un estabilizador de sistemas eléctricos de potencia adaptativo predictivo experto. Tesis doctoral. Universidad Nacional de Educación a Distancia, Escuela Técnica Superior de Ingenieros Industriales, Departamento de Ingeniería Eléctrica, Electrónica y de Control. Recuperado el diciembre de 2016 de: http://oa.upm.es/42/1/09200302.pdf.

Quingatuña, C. F. (2009). Estabilidad y Amortiguamiento de Oscilaciones en Sistemas Eléctricos con Alta Penetración Eólica. Tesis doctoral. Universidad Carlos III de Madrid, Departamento de Ingeniería Eléctrica, Electrónica y Automática. Recuperado el 2017, de http://orff.uc3m.es/bitstream/handle/10016/6731/ CarlosGallardoTesisdoctoral.pdf?sequence $=1$.

Sánchez, H. M., Pérez, A. J., \& Castrillon, G. N. (2012). Metodologías Heurísticas para ajuste de PSS. Jornadas técnicas ISA. Obtenido de 
https://es.scribd.com/document/236943946/Meto dologias-Heuristicas-Para-Ajuste-de-Pss

Verdejo, H., Gonzalez, D., Delpiano, J., \& Becker, C. (2015). Tuning of Power System Stabilizers using Multiobjective Optimization NSGA II. IEEE Latin America Transactions, 13(8):2653-2660. doi:10.1109/TLA.2015.7332145

Zea, A. Á. (2012). Amortiguamiento de Oscilaciones de Muy Baja Frecuencia Usando PSS's Multibanda con Señales Globales. Tesis ingeniería. Universidad Nacional de Colombia . Recuperado el Diciembre de 2016, de http://www.bdigital.unal.edu.co/5980/2/7108503. 2012.pdf.

\section{BIOGRAFÍAS}

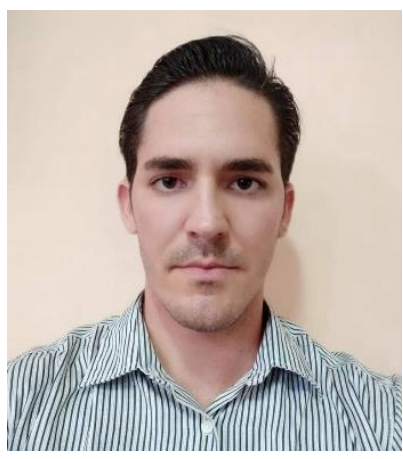

Ernesto García Hidalgo. Ingeniero electricista, Especialista de Desarrollo en Redes y Sistemas en la Unión Eléctrica. Participó en la XIX Convención Científica de Ingeniería y Arquitectura, La Habana, Cuba en su edición XVIII y XIX, $12^{\text {do }}$ Congreso Internacional de Educación Superior. Eléctrica, Cuba.

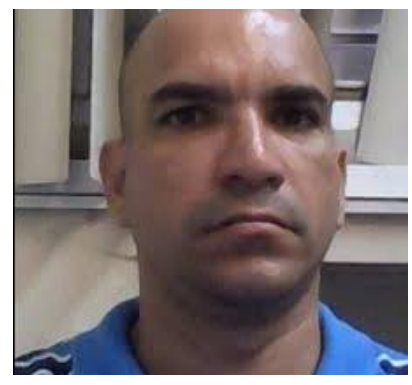

Maykop Pérez Martínez. Ingeniero electricista, Máster en Ingeniería Eléctrica, Profesor auxiliar, Centro de Investigaciones y Pruebas Electroenergética, CIPEL, Facultad de ingeniería eléctrica de la Universidad Tecnológica de la Habana, , Cuba. Jefe de disciplina de Circuitos Eléctricos. Misión Internacionalista Republica de Angola, participó $2^{\text {das }}$ jornadas Técnicos Científicas, Universidade Mandume Ya Ndemafuyo Escola Superior Politécnica do Namibe, XIX Convención Científica de Ingeniería y Arquitectura, La Habana, Cuba, Universidad $202012^{\text {do }}$ congreso internacional de educación superior, XVIII convención y feria internacional informática 2020 y en el III Congreso virtual argentino e Iberoamericano de tecnología y educación - COVAITE 2020, Asociación de Pedagogos de Cuba, XII taller internacional "Maestro ante los retos del siglos XXI", Congreso internacional Pedagogía 2021, IV taller de didáctica y aplicación de las ciencias básicas 2021 y VI taller internacional de formación básica y de postgrado en la universidad contemporánea.

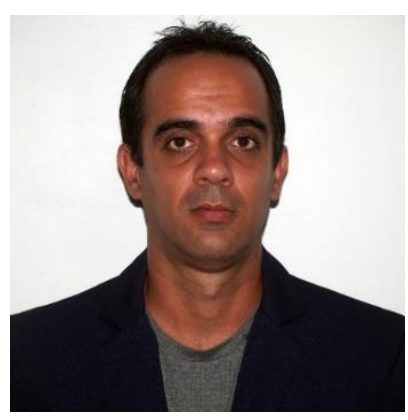

Yandry Rodríguez

Domínguez. Ingeniero

Electricista, Máster en Ingeniería Eléctrica y Doctor en ciencias técnicas, Profesor asistente y Vicedecano Docente, Centro de Investigaciones y Pruebas Electroenergética, CIPEL, Universidad Tecnológica de La Habana José Antonio Echeverría, La Habana, Cuba. Participó XIX Convención Científica de Ingeniería y Arquitectura, La Habana, Cuba. 